\title{
Existence Result for Fractional Klein-Gordon-Maxwell System with Quasicritical Potential Vanishing at Infinity
}

\author{
Canlin Gan, Ting Xiao, Qiongfen Zhang* \\ College of Science, Guilin University of Technology, Guilin, China \\ Email: gclmath@163.com, xiaotingLE@163.com, ^qfzhangcsu@163.com
}

How to cite this paper: Gan, C.L., Xiao, T. and Zhang, Q.F. (2020) Existence Result for Fractional Klein-Gordon-Maxwell System with Quasicritical Potential Vanishing at Infinity. Journal of Applied Mathematics and Physics, 8, 1318-1327.

https://doi.org/10.4236/jamp.2020.87101

Received: June 23, 2020

Accepted: July 19, 2020

Published: July 22, 2020

\section{Copyright $\odot 2020$ by author(s) and} Scientific Research Publishing Inc. This work is licensed under the Creative Commons Attribution International License (CC BY 4.0).

http://creativecommons.org/licenses/by/4.0/

\begin{abstract}
The following fractional Klein-Gordon-Maxwell system is studied $\left\{\begin{array}{ll}(-\Delta)^{p} u+V(x) u-(2 \omega+\phi) \phi u=K(x) f(u), & \text { in } \mathbb{R}^{3}, \\ (\Delta)^{p} \phi=(\omega+\phi) u^{2}, & \text { in } \mathbb{R}^{3},\end{array}\right.$ where $p \in(3 / 4,1)$, $(-\Delta)^{p}$ stands for the fractional Laplacian, $\omega>0$ is a constant, $V$ is vanishing potential and $K$ is a smooth function. Under some suitable conditions on $K$ and $f$, we obtain a Palais-Smale sequence by using a weaker Ambrosetti-Rabinowitz condition and prove the ground state solution for this system by employing variational methods. In particular, this kind of problem is a vast range of applications and challenges.
\end{abstract}

\section{Keywords}

Vanishing Potential, Fractional Klein-Gordon-Maxwell System, Variational Methods, Ground State Solution

\section{Introduction}

In this paper, the following fractional Klein-Gordon-Maxwell system is considered

$$
\begin{cases}(-\Delta)^{p} u+V(x) u-(2 \omega+\phi) \phi u=K(x) f(u), & \text { in } \mathbb{R}^{3}, \\ (\Delta)^{p} \phi=(\omega+\phi) u^{2}, & \text { in } \mathbb{R}^{3},\end{cases}
$$

where $p \in(3 / 4,1),(-\Delta)^{p}$ denotes the fractional Laplacian operator, $V$ is zero mass potential and $K$ is a smooth function. When $(2 \omega+\phi) \phi u=0$, system (1.1) reduces to a fractional Schrödinger equation. The fractional Schrödinger equation was first proposed by Laskin [1] [2] as a result of expanding the Feynman 
path integral from the Brownian-like to the Lévy-like quantum mechanical paths. This kind of problem can apply to various fields. For example, Li et al. [3] studied a class of fractional Schrödinger equation with potential vanishing at infinity by using variational methods and obtained a positive solution for this equation. For more results about fractional Schrödinger equation, please see [4] [5] [6] and the references therein.

If $p=1, V(x)=m^{2}-\omega^{2}$ and $K(x) f(u)=|u|^{q-2} u$, system (1.1) reduces to a Klein-Gordon-Maxwell equation, which was first studied by Benci and Fortunato [7] as a model describing a nonlinear Klein-Gordon equation interacting with an electromagnetic field with $4<q<6$. For more details on the physical aspects of this problem, we refer the readers to see [8] and references therein. When $2<q<4$ and $0<\omega<\sqrt{\frac{q}{2}-1} m$, D'Aprile and Mugnai [9] investigated the following system

$$
\left\{\begin{array}{l}
-\Delta u+\left[m^{2}-(\omega+\phi)^{2}\right] \phi u=|u|^{q-2} u, \quad x \in \mathbb{R}^{3}, \\
\Delta \phi=(\omega+\phi) u^{2}, \quad x \in \mathbb{R}^{3},
\end{array}\right.
$$

they obtained some results which complete the results obtained in [7].

In recent years, under various hypotheses on the potential $V(x)$ and the nonlinearity $f(u)$, the existence of positive, multiple, ground state solutions for Klein-Gordon-Maxwell systems or similar systems, has been widely studied in the literature. For example, Azzollini and Pomponio [10] first proved the existence of a ground state solution for system (1.2) when the nonlinearity is more general. He [11] first considered a Klein-Gordon-Maxwell system with non-constant potential. Li and Tang [12] improved the result of [11]. A nonlinear Klein-Gordon-Maxwell system with sign-changing potential was first considered by Ding and Li in [13]. They obtained infinitely many solutions by symmetric mountain pass theorem. Otherwise, there are many works about the nonhomogeneous Klein-Gordon-Maxwell system. Wang [14] proved that a nonhomogeneous Klein-Gordon-Maxwell system had two solutions. In [15], Gan et al. obtained two solutions for a type of nonhomogeneous Klein-Gordon-Maxwell system with sign-changing potential. Another example is [16], Miyagaki et al. investigated system (1.1) with fractional Laplacian and $f$ satisfied the following type of Ambrosetti-Rabinowitz condition:

(H4') For all $u>0$, There exists $\mu>4$ such that $0<\mu F(u) \leq f(u) u$, where $F(u)=\int_{0}^{u} f(t) \mathrm{d} t$.

Inspired mainly by the aforementioned results, we find a ground state solution for (1.1) with potential vanishing at infinity. To show our result, we make the following assumptions first:

(H1) $V \in C\left(\mathbb{R}^{3},(0,+\infty)\right), \quad K \in L^{\infty}\left(\mathbb{R}^{3}\right) \cap C\left(\mathbb{R}^{3},(0,+\infty)\right)$ and

$$
K / V \in L^{\infty}\left(\mathbb{R}^{3}\right),
$$

or for any $p \in(0,1)$, there exists $s \in\left(2,2_{p}^{*}\right)$, where $2_{p}^{*}=6 /(3-2 p)$, such that 


$$
\lim _{|x| \rightarrow \infty} \frac{K(x)}{V(x)^{\gamma}}=0, \quad \gamma=\frac{2 p s-3(s-2)}{4 p} \in(0,1) .
$$

(H2) $f \in C\left(\mathbb{R}, \mathbb{R}^{+}\right)$and $\left.f\right|_{\mathbb{R}^{-}}=0$.If $(1.3)$ holds, then

$$
\limsup _{t \rightarrow 0^{+}} \frac{f(t)}{t}=0 \text {. }
$$

If (1.4) holds, then

$$
\limsup _{t \rightarrow 0^{+}} \frac{f(t)}{t^{s-1}}<+\infty \text {. }
$$

(H3) If (1.3) holds, then

$$
\limsup _{t \rightarrow+\infty} \frac{f(t)}{t^{2_{p}^{*}-1}}=0
$$

If condition (1.4) holds, we assume that

$$
\limsup _{s \rightarrow+\infty} \frac{F(t)}{t^{p}}<+\infty .
$$

(H4) There exists $\mu>2$, such that $f(u) u \geq \mu F(u)>0$ for all $u>0$.

To the best of our knowledge, Ambrosetti-Rabinowitz condition (AR condition for short) plays an important role in proving the boundedness of Palais-Smale sequence (PS sequence for short). In recent years, there are many papers devoted to replacing (AR) condition with weaker condition. It is easy to see that (H4) is weaker than (H4'). In this paper, we obtain a (PS) sequence by using the weaker (AR) condition. Besides, it seems that there is only one work about the Klein-Gordon-Maxwell system involving fractional Laplacian.

Theorem 1.1. Assume that $p \in(3 / 4,1)$ and (H1)-(H4) hold. Then problem (1.1) admits a positive solution in $E$, where $E$ is defined in Section 2.

In this paper, the main difficulty is lack of compactness of Sobolev embedding in whole space because of the nonlocal term $\phi$ and the fractional operator. To overcome this problem, we use the reduction method introduced by Caffarelli and Silvestre [17] and recover the compactness by the interaction of the behaviour of the potential and nonlinearity.

This paper is organized as follows. In Section 2, some preliminary results are presented. In Section 3, we give the proof of main result.

\section{Preliminaries}

In this section, by the local reduction derived from Caffarelli and Silvestre [17], we first reformulate the nonlocal fractional system (1.1) into a local system, that is

$$
\begin{cases}-\operatorname{div}\left(y^{1-2 p} \nabla w_{1}\right)=0, & \text { in } \mathbb{R}_{+}^{4}, \\ w_{1}=u, & \text { on } \mathbb{R}^{3} \times\{0\}, \\ k_{p} y^{1-2 p} \frac{\partial w_{1}}{\partial \eta}=K(x) f(u)+(2 \omega+\phi) \phi u-V(x) u, & \text { on } \mathbb{R}^{3} \times\{0\}, \\ -\operatorname{div}\left(y^{1-2 p} \nabla w_{2}\right)=0, & \text { in } \mathbb{R}_{+}^{4}, \\ w_{2}=\phi, & \text { on } \mathbb{R}^{3} \times\{0\}, \\ k_{p} y^{1-2 p} \frac{\partial w_{2}}{\partial \eta}=(\omega+\phi) u^{2}, & \text { on } \mathbb{R}^{3} \times\{0\},\end{cases}
$$


where $\operatorname{div}\left(y^{1-2 p} \nabla w_{1}\right)$ denotes the divergence of $y^{1-2 p} \nabla w_{1}$ and $k_{p}=2^{1-2 p} \Gamma(1-p) / \Gamma(p)$ such that

$$
-k_{p} \lim _{y \rightarrow 0^{+}} y^{1-2 p} \frac{\partial w_{1}(x, y)}{\partial y}=(-\Delta)^{p} u(x),
$$

where $\phi(x)=w_{2}(x, 0):=\tilde{w}_{2}, u(x)=w_{1}(x, 0):=\tilde{w}_{1}$, and

$$
y^{1-2 p} \frac{\partial w_{1}}{\partial \eta}=-\lim _{y \rightarrow 0^{+}} y^{1-2 p} \frac{\partial w_{1}}{\partial y},
$$

is the outward normal derivative of $w_{1}$. Similar definition is given for $w_{2}$.

For $p \in(3 / 4,1)$ and $\phi: \mathbb{R}^{3} \rightarrow \mathbb{R}$, the fractional Laplacian $(-\Delta)^{p}$ of $\phi$ is defined by

$$
\mathcal{F}\left((-\Delta)^{p} \varphi\right)(z)=|z|^{2 p} \mathcal{F}(\varphi)(z), \quad z \in \mathbb{R}^{3},
$$

where $\mathcal{F}$ denotes the Fourier transform, that is

$$
\mathcal{F}(\varphi)(z)=\frac{1}{(2 \pi)^{3 / 2}} \int_{\mathbb{R}^{3}} \exp (-2 \pi j z \cdot x) \varphi(x) \mathrm{d} x,
$$

where $j$ denotes the imaginary unit. When $\varphi$ is smooth enough, the $(-\Delta)^{p}$ of $\varphi$ can be obtained by the following singular integral

$$
(-\Delta)^{p} \varphi(x)=c_{\alpha} P . V \cdot \int_{\mathbb{R}^{3}} \frac{\varphi(x)-\varphi(y)}{|x-y|^{3+2 p}} \mathrm{~d} y, \quad x \in \mathbb{R}^{3},
$$

where $c_{\alpha}$ is a normalization constant and P.V. is the principle value.

For any $p \in(3 / 4,1), \quad X^{2 p}\left(\mathbb{R}_{+}^{4}\right)$ and $H^{p}\left(\mathbb{R}^{3}\right)$ are the completion of $C_{0}^{\infty}\left(\overline{\mathbb{R}_{+}^{4}}\right)$ and $C_{0}^{\infty}\left(\mathbb{R}^{3}\right)$, and endowed with the norms

$$
\begin{gathered}
\|u\|_{X^{2 p}}:=\left(\int_{\mathbb{R}_{+}^{4}} k_{p} y^{1-2 p}|\nabla u|^{2} \mathrm{~d} x \mathrm{~d} y\right)^{1 / 2}, \\
\|u\|_{H^{p}}:=\left(\int_{\mathbb{R}^{3}}|2 \pi z|^{2 p}|\mathcal{F}(u(z))|^{2} \mathrm{~d} z\right)^{1 / 2}=\left(\int_{\mathbb{R}^{3}}\left|(-\Delta)^{p} u\right|^{2} \mathrm{~d} x\right)^{1 / 2},
\end{gathered}
$$

respectively. The Sobolev space $D^{p, 2}\left(\mathbb{R}_{+}^{4}\right)$ is defined by

$$
D^{p, 2}\left(\mathbb{R}_{+}^{4}\right)=\left\{u \in L^{2^{*}}\left(\mathbb{R}_{+}^{4}\right):|z|^{p} \hat{u} \in L^{2}\left(\mathbb{R}_{+}^{4}\right)\right\},
$$

which is the completion of $C_{0}^{\infty}\left(\mathbb{R}_{+}^{4}\right)$ under the norm

$$
\|u\|_{D^{p, 2}\left(\mathbb{R}_{+}^{4}\right)}^{2}=\left\|(-\Delta)^{p / 2} u\right\|_{2}^{2}=\int_{\mathbb{R}_{+}^{4}} y^{1-2 p}|\nabla(u)|^{2} \mathrm{~d} x \mathrm{~d} y, \quad u \in D^{p, 2}\left(\mathbb{R}_{+}^{4}\right) .
$$

Let $E$ be defined by

$$
E=\left\{u \in X^{2 p}\left(\mathbb{R}_{+}^{4}\right): \int_{\mathbb{R}^{3}} V(x) u(x, 0)^{2} \mathrm{~d} x<\infty\right\},
$$

which is endowed with norm

$$
\|u\|:=\left(\int_{\mathbb{R}_{+}^{4}} k_{p} y^{1-2 p}|\nabla u|^{2} \mathrm{~d} x \mathrm{~d} y+\int_{\mathbb{R}^{3}} V(x) u(x, 0)^{2} \mathrm{~d} x\right)^{1 / 2},
$$

then $E$ is a Hilbert space. In the following, for convenience, for any $u$, let $\tilde{u}:=u(x, 0)$. 
The functional associated to $(2.1)$ is given by

$$
\begin{aligned}
\Phi\left(w_{1}\right)= & \frac{k_{p}}{2} \int_{\mathbb{R}_{+}^{4}} y^{1-2 p}\left|\nabla w_{1}\right|^{2} \mathrm{~d} x \mathrm{~d} y+\frac{1}{2} \int_{\mathbb{R}^{3}} V(x) \tilde{w}_{1}^{2} \mathrm{~d} x-\frac{1}{2} \int_{\mathbb{R}^{3}} \omega \tilde{w}_{2} \tilde{w}_{1}^{2} \mathrm{~d} x \\
& -\int_{\mathbb{R}^{3}} K(x) F\left(\tilde{w}_{1}\right) \mathrm{d} x, \quad w_{1} \in E,
\end{aligned}
$$

which is of $C^{1}$ by (H1)-(H3).

A vector $w_{1}$ is a weak solution of system (2.1) if $\left\langle\Phi^{\prime}\left(w_{1}\right), U\right\rangle=0$ for any $U \in E$, i.e.

$$
\begin{aligned}
\left\langle\Phi^{\prime}\left(w_{1}\right), U\right\rangle= & k_{p} \int_{\mathbb{R}_{+}^{4}} y^{1-2 p}\left\langle\nabla w_{1}, \nabla U\right\rangle \mathrm{d} x \mathrm{~d} y+\int_{\mathbb{R}^{3}} V(x) \tilde{w}_{1} \tilde{U} \mathrm{~d} x \\
& -\int_{\mathbb{R}^{3}}\left[2 \omega+\tilde{w}_{2}\right] \tilde{w}_{2} \tilde{w}_{1} \tilde{U} \mathrm{~d} x-\int_{\mathbb{R}^{3}} K(x) f\left(\tilde{w}_{1}\right) \tilde{U} \mathrm{~d} x .
\end{aligned}
$$

Lemma 2.1. [16] For every $u(x, y) \in X^{2 p}\left(\mathbb{R}_{+}^{4}\right)$, there exists a unique $\phi=\phi_{u}(x, y) \in D^{p, 2}\left(\mathbb{R}_{+}^{4}\right)$ which solves

$$
\begin{cases}-\operatorname{div}\left(y^{1-2 p} \nabla w\right)=0, & \text { in } \mathbb{R}_{+}^{4}, \\ k_{p} y^{1-2 p} \frac{\partial w}{\partial \eta}=(\omega+\phi) u^{2}, & \text { on } \mathbb{R}^{3} \times\{0\} .\end{cases}
$$

Furthermore, in the set $\{(x, 0): \tilde{u}:=u(x, 0) \not \equiv 0\}$, we have $-\omega \leq \phi_{u} \leq 0$ for $\omega>0$.

Let the weighted Banach space be

$$
L_{K}^{s}=\left\{u: \mathbb{R}_{+}^{4} \rightarrow \mathbb{R} \text { is measurable and } \int_{\mathbb{R}^{3}} K(x)|\tilde{u}|^{s} \mathrm{~d} x<\infty\right\}, \quad s \in(1,+\infty)
$$

under the norm

$$
\|u\|_{L_{K}^{s}}=\left(\int_{\mathbb{R}^{3}} K(x)|\tilde{u}|^{s} \mathrm{~d} x\right)^{1 / s} .
$$

The following Proposition 2.2 comes from the arguments in [18].

Proposition 2.2. [18] Assume that (H1) holds. Then

1) $E \hookrightarrow L_{K}^{q}$ is compact for all $q \in\left(2,2_{p}^{*}\right)$, provided that (1.3) holds,

2) $E \hookrightarrow L_{K}^{s}$ is compact provided that (1.4) holds,

3) If $u_{k} \rightarrow u$ in $E$, then up to a subsequence

$$
\lim _{k \rightarrow \infty} \int_{\mathbb{R}^{3}} K(x) F\left(\tilde{u}_{k}\right) \mathrm{d} x=\int_{\mathbb{R}^{3}} K(x) F(\tilde{u}) \mathrm{d} x ;
$$

4) If $u_{k} \rightarrow u$ in $E$, then up to a subsequence

$$
\lim _{k \rightarrow \infty} \int_{\mathbb{R}^{3}} K(x) \tilde{u}_{k} f\left(\tilde{u}_{k}\right) \mathrm{d} x=\int_{\mathbb{R}^{3}} K(x) \tilde{u} f(\tilde{u}) \mathrm{d} x
$$

5) If $u_{k} \rightarrow u$ in $E$, then up to a subsequence, for any $z \in E$,

$$
\lim _{k \rightarrow \infty} \int_{\mathbb{R}^{3}} K(x) f\left(\tilde{u}_{k}\right) \tilde{z} \mathrm{~d} x=\int_{\mathbb{R}^{3}} K(x) f(\tilde{u}) \tilde{z} \mathrm{~d} x .
$$

Lemma 2.3. [16] If $u_{k}(x, y) \rightarrow u(x, y)$ in $E$, as $k \rightarrow \infty$, then passing to a subsequence if necessary, $\phi_{u_{k}}(x, y) \rightarrow \phi_{u}(x, y)$ weakly in $D^{s, 2}\left(\mathbb{R}_{+}^{4}\right)$, as $k \rightarrow \infty$.

Lemma 2.4. Assume that $(\mathrm{H} 2)$ and $(\mathrm{H} 3)$ hold. Then the functional $\Phi$ satisfies

1) There exists $\beta, \rho>0$ such that $\Phi(u) \geq \beta$ if $\|u\|=\rho$; 
2) There exists $u_{0} \in E \backslash\{0\}$ with $\|u\|>\rho$ such that $\Phi\left(u_{0}\right) \leq 0$.

The proof of Lemma 2.4 is standard, so we omit the details here.

From Lemma 2.4, there exists a $(P S)_{c}$ sequence $\left\{u_{k}\right\} \subset E$ such that

$$
\Phi\left(u_{k}\right) \rightarrow c \text { and }\left\|\Phi^{\prime}\left(u_{k}\right)\right\|\left(1+\left\|u_{k}\right\|\right) \rightarrow 0, \quad \text { as } k \rightarrow+\infty,
$$

where

$$
c=\inf _{\gamma \in \Gamma} \max _{t \in[0,1]} \Phi(\gamma(t))
$$

with

$$
\Gamma=\{\gamma \in C([0,1], E) ; \gamma(0)=0 \text { and } \Phi(\gamma(1)) \leq 0\} .
$$

\section{Proof of Main Result}

Lemma 3.1. Assume that $(\mathrm{H} 2)-(\mathrm{H} 4)$ hold. Then the $(P S)_{c}$ sequence $\left\{u_{k}\right\} \subset E$ given in (2.6) is bounded.

Proof. Let $\left\{u_{k}\right\} \subset E$ be a $(P S)_{c}$ sequence of $\Phi$. Arguing indirectly, suppose $\left\|u_{k}\right\| \rightarrow \infty$ such that

$$
\Phi\left(u_{k}\right) \rightarrow c, \quad \Phi^{\prime}\left(u_{k}\right) \rightarrow 0, \quad \text { as } k \rightarrow \infty,
$$

after passing to a subsequence. Denote $v_{k}:=u_{k} /\left\|u_{k}\right\|$. Then $\left\|v_{k}\right\|=1, v_{k} \rightarrow v_{0}$ in $E$ and $v_{k}(x) \rightarrow v_{0}(x)$ for a.e. $x \in \mathbb{R}^{3}$. If $v_{0}=0$, by the fact $v_{k} \rightarrow 0$ in $L^{2}\left(\mathbb{R}^{3}\right)$, (2.2), (2.3), (2.4), (3.1) and Lemma 2.1, there are two cases to consider.

Case (1): $\mu \in[4, \infty)$. From (2.2), (2.3), (2.4) and (3.1), we derive

$$
\begin{aligned}
o(1)= & \frac{\mu \Phi\left(u_{k}\right)-\left\langle\Phi^{\prime}\left(u_{k}\right), u_{k}\right\rangle}{\left\|u_{k}\right\|^{2}} \\
= & \left(\frac{\theta}{2}-1\right)+\left(2-\frac{\mu}{2}\right) \int_{\mathbb{R}^{3}} \frac{\omega \phi_{u_{k}}(x, 0) \tilde{u}_{k}^{2}}{\left\|u_{k}\right\|^{2}} \mathrm{~d} x+\int_{\mathbb{R}^{3}} \frac{\phi_{u_{k}}^{2}(x, 0) \tilde{u}_{k}^{2}}{\left\|u_{k}\right\|^{2}} \mathrm{~d} x \\
& +\int_{\mathbb{R}^{3}} \frac{K(x)\left[f\left(\tilde{u}_{k}\right) \tilde{u}_{k}-\mu F\left(\tilde{u}_{k}\right)\right]}{\left\|u_{k}\right\|^{2}} \mathrm{~d} x \\
\geq & \frac{\mu}{2}-1+o(1),
\end{aligned}
$$

then $0 \geq \frac{\mu}{2}-1$, which contradict $\mu \geq 4$.

Case (2): $\mu \in(2,4)$. In this case, by (2.2), (2.3), (2.4), (3.1) and Lemma 2.1, one gets

$$
\begin{aligned}
o(1) & =\frac{\mu \Phi\left(u_{k}\right)-\left\langle\Phi^{\prime}\left(u_{k}\right), u_{k}\right\rangle}{\left\|u_{k}\right\|^{2}} \\
& \geq\left(\frac{\mu}{2}-1\right)+\left(2-\frac{\mu}{2}\right) \int_{\mathbb{R}^{3}} \frac{\omega \phi_{u_{k}}(x, 0) \tilde{u}_{k}^{2}}{\left\|u_{k}\right\|^{2}} \mathrm{~d} x \\
& \geq\left(\frac{\mu}{2}-1\right)-\left(2-\frac{\mu}{2}\right) \omega^{2}\left|v_{k}\right|_{2}^{2} \\
& =\frac{\theta}{2}-1+o(1),
\end{aligned}
$$


then $0 \geq \frac{\mu}{2}-1$, which contradict $\mu>2$.

If $v_{0} \neq 0$, then meas $\left\{\Omega_{1}\right\}>0$, where $\Omega_{1}:=\left\{x \in \mathbb{R}^{3}: v_{0}(x, 0) \neq 0\right\}$. For $x \in \Omega_{1}$, we have $\left|\tilde{u}_{k}\right| \rightarrow \infty$ as $k \rightarrow \infty$, and then, from (H4), we get

$$
\frac{F\left(\tilde{u}_{k}\right)}{\tilde{u}_{k}^{2}} \tilde{v}_{k}^{2} \rightarrow+\infty, \quad \text { as } k \rightarrow \infty \text {. }
$$

From (3.2) and Fatou's Lemma, we obtain

$$
\int_{\Omega_{1}} \frac{F\left(\tilde{u}_{k}\right)}{\tilde{u}_{k}^{2}} \tilde{v}_{k}^{2} \mathrm{~d} x \rightarrow+\infty, \quad \text { as } k \rightarrow \infty .
$$

From (2.2), (2.3), (3.1), (3.3) and Lemma 2.1, we have

$$
\begin{aligned}
0 & =\lim _{k \rightarrow \infty} \frac{\Phi\left(u_{k}\right)}{\left\|u_{k}\right\|^{2}} \\
& =\lim _{k \rightarrow \infty}\left[\frac{1}{2}-\frac{1}{2} \int_{\mathbb{R}^{3}} \frac{\omega \phi_{u_{k}}(x, 0) \tilde{u}_{k}^{2}}{\left\|u_{k}\right\|^{2}} \mathrm{~d} x-\int_{\mathbb{R}^{3}} \frac{K(x) F\left(\tilde{u}_{k}\right)}{\left\|u_{k}\right\|^{2}} \mathrm{~d} x\right] \\
& =\frac{1}{2}+o(1)-\lim _{k \rightarrow \infty} \int_{\mathbb{R}^{3}} \frac{K(x) F\left(\tilde{u}_{k}\right)}{\left\|u_{k}\right\|^{2}} \mathrm{~d} x \\
& \leq \frac{1}{2}+o(1)-\lim _{k \rightarrow \infty} \int_{\Omega_{1}} \frac{K(x) F\left(\tilde{u}_{k}\right)}{\tilde{u}_{k}^{2}} \tilde{v}_{k}^{2} \mathrm{~d} x \\
& =-\infty,
\end{aligned}
$$

a contradiction. Hence, the boundedness of $\left\{u_{n}\right\}$ in $E$ is obtained.

Proof of Theorem 1.1. Let $\left\{u_{k}\right\}$ be a $(P S)_{c}$ sequence given in (2.6). It follows from Lemma 3.1 that $\left\{u_{k}\right\}$ is bounded, passing to a subsequence, one can assume that there is $u \in E$ such that

$$
u_{k} \rightarrow u \text {, weakly in } E \text {, as } k \rightarrow \infty \text {. }
$$

It suffices to show that $u_{k} \rightarrow u$, as $k \rightarrow \infty$. By Proposition 2.2, one has

$$
\lim _{k \rightarrow \infty} \int_{\mathbb{R}^{3}} K(x) f\left(\tilde{u}_{k}\right) \tilde{u}_{k} \mathrm{~d} x=\int_{\mathbb{R}^{3}} K(x) f(\tilde{u}) \tilde{u} \mathrm{~d} x .
$$

From (2.4), we have

$$
\begin{aligned}
\left\langle\Phi^{\prime}\left(u_{k}\right), U\right\rangle= & k_{s} \int_{\mathbb{R}_{+}^{4}} y^{1-2 s}\left\langle\nabla u_{k}, \nabla U\right\rangle \mathrm{d} x \mathrm{~d} y+\int_{\mathbb{R}^{3}} V(x) \tilde{u}_{k} \tilde{U} \mathrm{~d} x \\
& -\int_{\mathbb{R}^{3}}\left[2 \omega+\tilde{w}_{2}\right] \tilde{w}_{2} \tilde{u}_{k} \tilde{U} \mathrm{~d} x-\int_{\mathbb{R}^{3}} K(x) f\left(\tilde{u}_{k}\right) \tilde{U} \mathrm{~d} x .
\end{aligned}
$$

By $\left\langle\Phi^{\prime}\left(u_{k}\right), u_{k}\right\rangle=o_{n}(1)$, one gets

$$
\lim _{k \rightarrow \infty}\left\|u_{k}\right\|^{2}=\lim _{k \rightarrow \infty}\left[\int_{\mathbb{R}^{3}}\left(2 \omega+\tilde{w}_{2}\right) \tilde{w}_{2} \tilde{u}_{k}^{2} \mathrm{~d} x-\int_{\mathbb{R}^{3}} K(x) f\left(\tilde{u}_{k}\right) \tilde{u}_{k} \mathrm{~d} x\right] .
$$

By Proposition 2.2, one obtains

$$
\lim _{k \rightarrow \infty} \int_{\mathbb{R}^{3}} K(x) f\left(\tilde{u}_{k}\right) \tilde{u} \mathrm{~d} x=\int_{\mathbb{R}^{3}} K(x) f(\tilde{u}) \tilde{u} \mathrm{~d} x .
$$

From the proof of Lemma 2.3 in [16], we know that there exists a $z \in D^{p, 2}\left(\mathbb{R}_{+}^{4}\right)$ such that

$$
\phi_{u_{k}} \rightarrow z \text { in } L^{r}\left(\mathbb{R}^{3} \times\{0\}\right) \text { as } k \rightarrow \infty \text {, }
$$




$$
\phi_{u_{k}} \rightarrow z \text { in } L_{l o c}^{r}\left(\mathbb{R}^{3} \times\{0\}\right) \text { as } k \rightarrow \infty, r \in[2,6 /(3-2 p)] \text {, }
$$

and $\phi_{u}=z$. Hence, from Lemma 2.3, we obtain that

$$
\lim _{n \rightarrow \infty} \int_{\mathbb{R}^{3}}\left(2 \omega+\tilde{w}_{2}\right) \tilde{w}_{2} \tilde{u}_{k}^{2} \mathrm{~d} x=\int_{\mathbb{R}^{3}}(2 \omega+\tilde{z}) \tilde{z} \tilde{u}^{2} \mathrm{~d} x .
$$

Then

$$
\lim _{n \rightarrow \infty}\left\|u_{k}\right\|^{2}=\int_{\mathbb{R}^{3}}(2 \omega+\tilde{z}) \tilde{z} \tilde{u}^{2} \mathrm{~d} x+\int_{\mathbb{R}^{3}} K(x) f(\tilde{u}) \tilde{u} \mathrm{~d} x .
$$

Otherwise, since $\left\langle\Phi^{\prime}(u), u\right\rangle=o(1)$, one has

$$
\|u\|^{2}=\int_{\mathbb{R}^{3}}(2 \omega+\tilde{z}) \tilde{z} \tilde{u}^{2} \mathrm{~d} x+\int_{\mathbb{R}^{3}} K(x) f(\tilde{u}) \tilde{u} \mathrm{~d} x .
$$

Hence, from (3.7) and (3.8), we have

$$
\lim _{n \rightarrow \infty}\left\|u_{k}\right\|^{2}=\|u\|^{2},
$$

which shows that

$$
u_{k} \rightarrow u \quad \text { in } E, \quad \text { as } k \rightarrow \infty .
$$

Hence, we conclude that

$$
\Phi(u)=c \text { and } \Phi^{\prime}(u)=0 .
$$

Thus, $u$ is a ground state solution for $\Phi$. It follows from $u_{k} \geq 0$ that $u \geq 0$. Since there is a $(P S)_{c}$ sequence $\left\{u_{k}\right\}$, we can obtain that $u$ is positive from Lemma 2.1 by contradiction.

\section{Conclusion}

In this paper, we first reformulated the system (1.1) into a local system by using the local reduction. Then, we take advantage of the interaction of the behaviour of the potential and nonlinearity to recover the compactness. Meanwhile, we obtained a Palais-Smale sequence by using a weaker Ambrosetti-Rabinowitz condition. Finally, the existence of positive solution is proved by the mountain pass theorem. Obviously, the weaker Ambrosetti-Rabinowitz condition has been successfully applied to find the solution of the fractional Klein-Gordon-Maxwell system with potential vanishing at infinity. We hope that this result can be widely used in other systems.

\section{Acknowledgements}

The authors would like to thank the referees for their useful suggestions which have significantly improved the paper.

\section{Conflicts of Interest}

No potential conflict of interest was reported by the authors.

\section{Funding}

This work is supported by the National Natural Science Foundation of China (No. 11961014, No. 61563013) and Guangxi Natural Science Foundation (2016 
GXNSFAA380082, 2018GXNSFAA281021).

\section{References}

[1] Laskin, N. (2000) Fractional Quantum Mechanics and Lévy Path Integrals. Physics Letters A, 268, 298-305. https://doi.org/10.1016/S0375-9601(00)00201-2

[2] Laskin, N. (2002) Fractional Schrödinger Equation. Physical Review E, 66, 56-108. https://doi.org/10.1103/PhysRevE.66.056108

[3] Li, Q.Q., Teng, K.M. and Wu, X. (2017) Existence of Positive Solutions for a Class of Critical Fractional Schrödinger Equations with Potential Vanishing at Infinity. Mediterranean Journal of Mathematics, 14, 80. https://doi.org/10.1007/s00009-017-0846-5

[4] Teng, K. (2015) Multiple Solutions for a Class of Fractional Schrödinger Equations in $\mathrm{R}^{\mathrm{N}}$. Nonlinear Analysis, 21, 76-86.

[5] Zhang, X., Zhang, B.L. and Repovš, D. (2016) Existence and Symmetry of Solutions for Critical Fractional Schrödinger Equations with Bounded Potentials. Nonlinear Analysis, 142, 48-68. https://doi.org/10.1016/j.na.2016.04.012

[6] Wang, Z. and Zhou, H. (2016) Radial Sign-Changing Solution for Fractional Schrödinger Equation. Discrete \& Continuous Dynamical Systems, 36, 499-508. https://doi.org/10.3934/dcds.2016.36.499

[7] Benci, V. and Fortunato, D. (2001) The Nonlinear Klein-Gordon Equation Coupled with the Maxwell Equations. Nonlinear Analysis, 47, 6065-6072.

https://doi.org/10.1016/S0362-546X(01)00688-5

[8] Benci, V. and Fortunato, D. (2002) Solitary Waves of the Nonlinear Klein-Gordon Equation Coupled with the Maxwell Equations. Reviews in Mathematical Physics, 14, 409-420. https://doi.org/10.1142/S0129055X02001168

[9] D’Aprile, T. and Mugnai, D. (2004) Solitary Waves of the Nonlinear Klein-GordonMaxwell and Schrödinger-Maxwell Equations. Proceedings of the Royal Society of Edinburgh Section A, 134, 893-906. https://doi.org/10.1017/S030821050000353X

[10] Azzollini, A. and Pomponio, A. (2010) Ground State Solutions for the Nonlinear Klein-Gordon-Maxwell Equations. Topological Methods in Nonlinear Analysis, 35, 33-42.

[11] He, X.M. (2014) Multiplicity of Solutions for a Nonlinear Klein-Gordon-Maxwell System. Acta Applicandae Mathematicae, 130, 237-250. https://doi.org/10.1007/s10440-013-9845-0

[12] Li, L. and Tang, C.L. (2014) Infinitely Many Solutions for a Nonlinear Klein-Gordon-Maxwell System. Nonlinear Analysis, 110, 157-169. https://doi.org/10.1016/j.na.2014.07.019

[13] Ding, L. and Li, L. (2014) Infinitely Many Standing Wave Solutions for the Nonlinear Klein-Gordon-Maxwell System with Sign-Changing Potential. Computers \& Mathematics with Applications, 68, 589-595. https://doi.org/10.1016/j.camwa.2014.07.001

[14] Wang, L.X. (2019) Two Solutions for a Nonhomogeneous Klein-Gordon-Maxwell System. Electronic Journal of Qualitative Theory of Differential Equations, 40, 1-12. https://doi.org/10.14232/ejqtde.2019.1.40

[15] Gan, C.L., Xiao, T. and Zhang, Q.F. (2020) Improved Results of Nontrivial Solutions for a Nonlinear Nonhomogeneous Klein-Gordon-Maxwell System Involving Sign-Changing Potential. Advances in Difference Equations, 167, 1-16. https://doi.org/10.1186/s13662-020-02634-9 
[16] Miyagaki, O.H., de Moura, E.L. and Ruviaro, R. (2019) Positive Ground State Solutions for Quasicritical the Fractional Klein-Gordon-Maxwell System with Potential Vanishing at Infinity. Complex Variables and Elliptic Equations, 64, 315-329. https://doi.org/10.1080/17476933.2018.1434625

[17] Caffarelli, L. and Silvestre, L. (2007) An Extension Problems Related to the Fractional Laplacian. Communications in Partial Differential Equations, 32, 1245-1260. https://doi.org/10.1080/03605300600987306

[18] Alves, C.O. and Souto, M.A.S. (2013) Existence of Solutions for a Class of Nonlinear Schrödinger Equations with Potential Vanishing at Infinity. Journal of Differential Equations, 254, 1977-1991. https://doi.org/10.1016/j.jde.2012.11.013 